\title{
Classical Hodgkin's lymphoma mixed cellularity type
}

\author{
Teiko Kawahigashi ${ }^{1}$ \\ ${ }^{1}$ Shonan Kamakura General Hospital
}

December 13, 2021

\begin{abstract}
An 81-year-old man presented to our hospital with a 6-month history of weight loss and lymphadenopathy. On examination, he had high fever and right axillary lymphadenopathy. A right axillary lymph-node excisional biopsy showed findings of mixed cellularity Hodgkin's lymphoma. However, he died before the results of the biopsy were obtained.
\end{abstract}

\section{Classical Hodgkin's lymphoma mixed cellularity type}

Teiko Kawahigashi, MD

Department of Internal Medicine, Ishigakijima Tokushukai Hospital

\section{Corresponding author:}

\section{Teiko Kawahigashi}

Department of Internal Medicine, Ishigakijima Tokushukai Hospital

446-1 Ohama, Ishigaki, Okinawa, 907-0001, Japan.

Telephone: +81-980-88-0123

Fax: +81-980-82-9511

E-mail address:teikokawahigashi@gmail.com

\section{CASE PRESENTATION}

An 81-year-old Japanese man with appetite and weight loss of unknown etiology was admitted to the emergency department. Over 6 months he lost $10 \mathrm{~kg}$, which was equivalent to $17 \%$ of his total body weight. Physical examination revealed fever (temperature, $38^{\circ} \mathrm{C}$ ) and multiple, firm, non-tender lymphadenopathies in the right axilla. Contrast-enhanced computed tomography showed mild splenomegaly and para-aortic lymphadenopathy (Figure 1). An excision biopsy of the axillary nodes showed Reed-Sternberg cells, confirming Hodgkin's lymphoma (HL) of the mixed cellularity type. Unfortunately, the patient died before a definite diagnosis could be made.

HL is a known curable lymphoid malignancy with high cure rates observed with multi-agent chemotherapy and often, radiation therapy. ${ }^{1}$ However, diagnosing HL in Japan is difficult because of its low incidence and nonspecific symptoms. ${ }^{1}$ Advanced age is a strong risk factor for malignancy in patients presenting with lymphadenopathy. The risk of malignancy in those aged $>40$ years is 10 times higher than that of those younger. ${ }^{2}$ This case is a reminder that malignancy should always be considered in elderly patients presenting with lymphadenopathy and B symptoms. A high index of suspicion for lymphoid malignancy can lead to prompt diagnosis, timely treatment, and better outcomes.

CONFLICT OF INTEREST 
No conflicts of interest in connection with this article.

\section{ACKNOWLEDGMENTS}

We would like to thank Editage (www.editage.jp) for English language editing.

\section{CONSENT}

Written informed consent was obtained from the patient's husband for publication of this case report and any accompanying images.

\section{REFERENCES}

Swerdlow, S. H., Campo, E., Harris, N. L., et al., editors. World Health Organization Classification of Tumours of Haematopoietic and Lymphoid Tissues. Lyon: IARC Press, 2008.

Emery, J. D., Gray, V., Walter, F. M., et al. 2017. The Improving Rural Cancer Outcomes Trial: a clusterrandomised controlled trial of a complex intervention to reduce time to diagnosis in rural cancer patients in Western Australia. British Journal of Cancer. 117(10):1459-1469.

Figure 1

Computed tomography showing multiple lymphadenopathies in the A) right axilla (arrow) and B) para-aortic area (arrow).

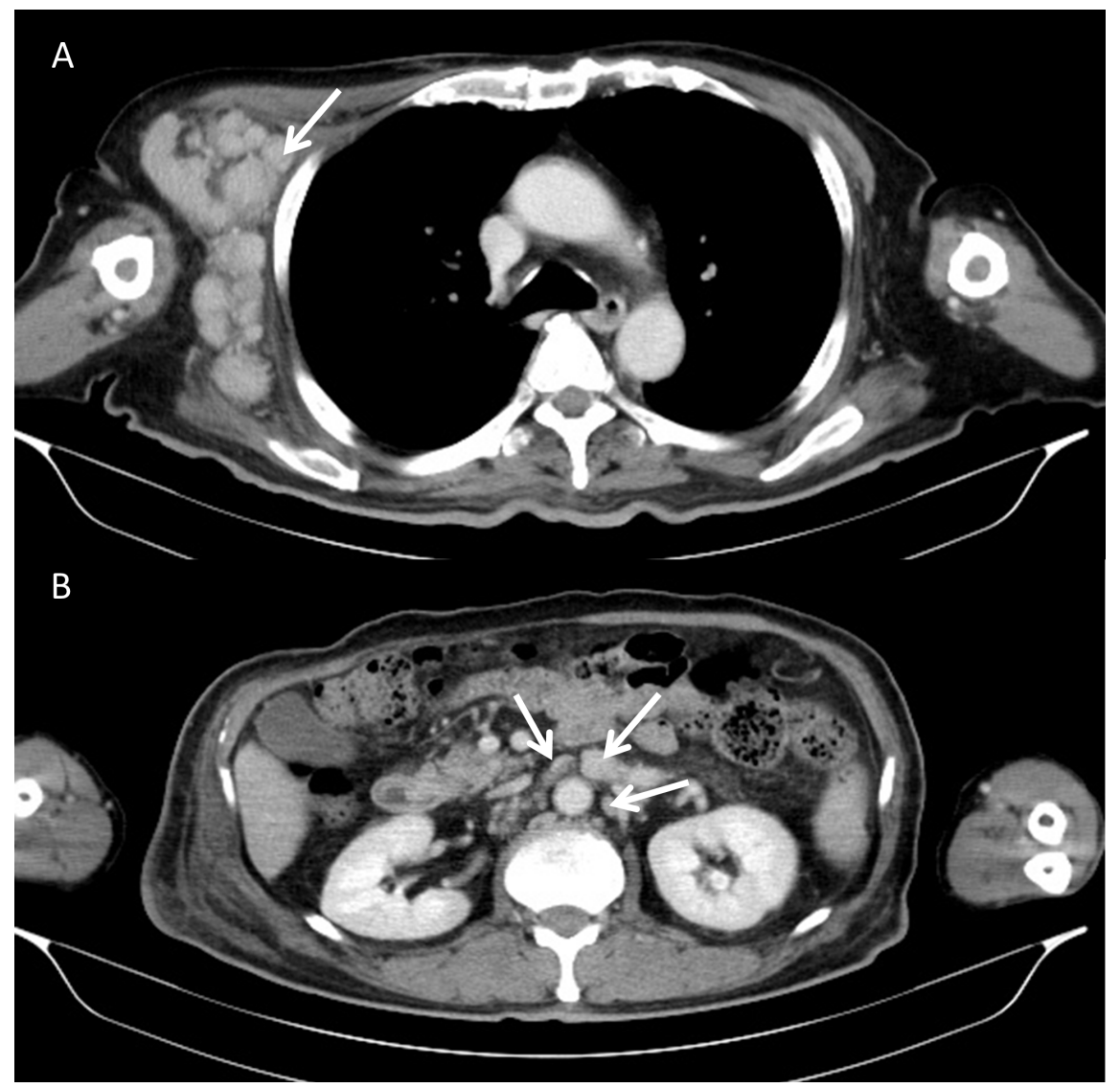

